\title{
$X$-band rf power production and deceleration in the two-beam test stand of the Compact Linear Collider test facility
}

\author{
E. Adli* \\ Department of Physics, University of Oslo, 0316 Oslo, Norway \\ R. Ruber and V. Ziemann \\ Department of Physics and Astronomy, Uppsala University, 75121 Uppsala, Sweden \\ R. Corsini, A. Dubrovskiy, and I. Syratchev \\ CERN, CH 1211 Geneva 23, Switzerland \\ (Received 26 May 2011; published 22 August 2011)
}

\begin{abstract}
We discuss $X$-band $\mathrm{rf}$ power production and deceleration in the two-beam test stand of the CLIC test facility at CERN. The rf power is extracted from an electron drive beam by a specially designed power extraction structure. In order to test the structures at high-power levels, part of the generated power is recirculated to an input port, thus allowing for increased deceleration and power levels within the structure. The degree of recirculation is controlled by a splitter and phase shifter. We present a model that describes the system and validate it with measurements over a wide range of parameters. Moreover, by correlating rf power measurements with the energy lost by the electron beam, as measured in a spectrometer placed after the power extraction structure, we are able to identify system parameters, including the form factor of the electron beam. The quality of the agreement between model and reality gives us confidence to extrapolate the results found in the present test facility towards the parameter regime of CLIC.
\end{abstract}

DOI: 10.1103/PhysRevSTAB.14.081001

PACS numbers: 29.20.Ej, 41.75.Ht, 41.20.Jb, 01.52.+r

\section{INTRODUCTION}

The Compact Linear Collider, CLIC [1], is a candidate for a multi-TeV linear collider. Reaching $\mathrm{TeV}$ energies within an acceptable length scale requires extremely high accelerating fields of $100 \mathrm{MV} / \mathrm{m}$ and an efficient distribution system for the microwaves that excite the fields inside the acceleration structures. Both points are addressed in the two-beam acceleration scheme for CLIC [2], where a moderate energy, but high-intensity electron beam excites electromagnetic fields inside so-called power extraction and transfer structures (PETS) which are then transferred to acceleration structures where a second beam is accelerated to $\mathrm{TeV}$ energies. In CLIC the current in the drive beam is on the order of 100 A to produce $100 \mathrm{MW}$ of microwave power at $12 \mathrm{GHz}$. The experimental verification of the two-beam acceleration scheme is the central task of the two-beam test stand (TBTS) [3] in the CLIC Test Facility 3 (CTF3) [4] at CERN. The CLIC machine is planned to have on the order of 100000 accelerating and PETS structures, and it is crucial to achieve a detailed understanding of the two-beam acceleration tests.

\footnotetext{
*Erik.Adli@fys.uio.no
}

Published by the American Physical Society under the terms of the Creative Commons Attribution 3.0 License. Further distribution of this work must maintain attribution to the author(s) and the published article's title, journal citation, and DOI.
In this paper we focus on the power production in the PETS structures on the drive beam side. In order to run the PETS structures at power levels required for CLIC, despite the fact that in CTF3 we only have beam currents of up to $30 \mathrm{~A}$, we employ a power recirculation scheme where a power splitter is employed that redirects part of the power generated in the PETS to the acceleration structures and the remaining part is directed via a phase shifter to the input of the PETS structures. In this way a resonant buildup of the power level inside the PETS structures to the desired $100 \mathrm{MW}$ level and beyond is achieved, allowing us to thoroughly test the PETS structures. A novelty of our resonant loop is that the power is continuously extracted from the drive beam. In order to better understand the measurements of the rf power levels and the energy loss of the drive beam that can be measured in a spectrometer beam line, we have constructed a recirculation model that permits one to determine with high precision relevant parameters of the combined system of the beam and rf fields. These include the power splitter and phase shifter settings, but more importantly the coupling of the beam to the fields inside the PETS, which depends strongly on the bunch length via the bunch form factor.

The detailed characterization of the TBTS power production presented in this paper is an important step towards full verification of the two-beam acceleration scheme, and furthermore the validity of the recirculation model we have 
developed will greatly add planning for future upgrades of the CLIC test facilities [5].

The paper is organized as follows. First, we briefly describe the drive beam generation and the TBTS. We then describe PETS rf power production with recirculation and we suggest a method to identify recirculation parameters. The method is then applied to different measurement series and, by comparing the measured power with the power reconstructed from the beam current measurements, we estimate the beam form factor. Finally, we correlate rf power extraction with deceleration measurements by calculating the total pulse energy loss using two different methods.

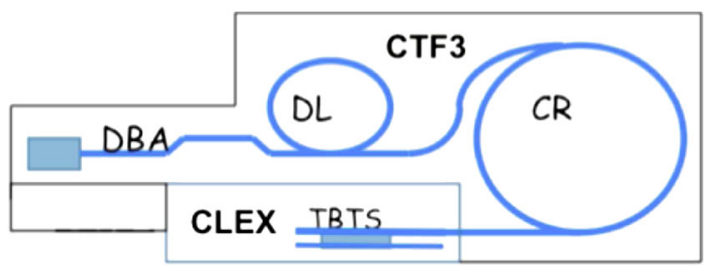

FIG. 1. Sketch of the CLIC Test Facility 3. An electron drive beam of about $4 \mathrm{~A}$ is accelerated by a fully loaded linac (DBA) with structures powered by compressed $3 \mathrm{GHz}$ rf pulses. The bunch frequency can be multiplied by a factor 2 in the delay loop (DL) and by a factor 4 in the combiner ring (CR) by interleaving bunches, for a maximum factor of 8 . The combined drive beam is then sent to the two-beam test stand located in the experimental area (CLEX). In CLEX an electron probe beam line is installed in parallel to the drive beam line.

\section{EXPERIMENTAL SETUP}

The rf power is extracted from an electron drive beam generated by the CTF3 drive beam complex [4], depicted in Fig. 1. A thermionic gun emits an average electron current of up to $4 \mathrm{~A}$ with a train length of $1.2 \mu \mathrm{s}$. The beam is bunched either by a $1.5 \mathrm{GHz}$ subharmonic buncher or a $3 \mathrm{GHz}$ buncher, depending on the mode of operation of CTF3. The bunch train is accelerated in a fully loaded linac, powered by $\mathrm{rf}$ power generated by $3 \mathrm{GHz}$ 30-40 MW klystrons subsequently compressed with LIPS pulse compressor systems [4]. This allows the drive beam to reach a maximum energy of about $150 \mathrm{MeV}$.

CTF3 is designed to generate a drive beam of about 30 A using a delay loop and a combiner ring. The delay loop can combine two $1.5 \mathrm{GHz}$ half-trains into one train combined by a factor 2 (double the intensity and frequency, half the train length). The combiner ring can further interleave the incoming bunch trains, increasing the frequency by a factor 4 . After compression the drive beam is sent into the CLIC experimental area, CLEX. Depending on the needs of the experiments, the beam sent to CLEX can be an uncombined beam (up to $4 \mathrm{~A}$ and $1.2 \mu \mathrm{s}$ ), combined by a factor 2 with the delay loop (up to $8 \mathrm{~A}$ and $0.6 \mu \mathrm{s}$ ) combined by a factor 4 with the combiner ring (up to $16 \mathrm{~A}$ and $300 \mathrm{~ns}$ ) or combined by a factor 8 , using both the delay loop and the combiner ring (up to $32 \mathrm{~A}$ and $150 \mathrm{~ns}$ ).

In CLEX the drive beam enters the TBTS where it is decelerated by a PETS structure. The TBTS also has a probe beam line in which a second beam is accelerated

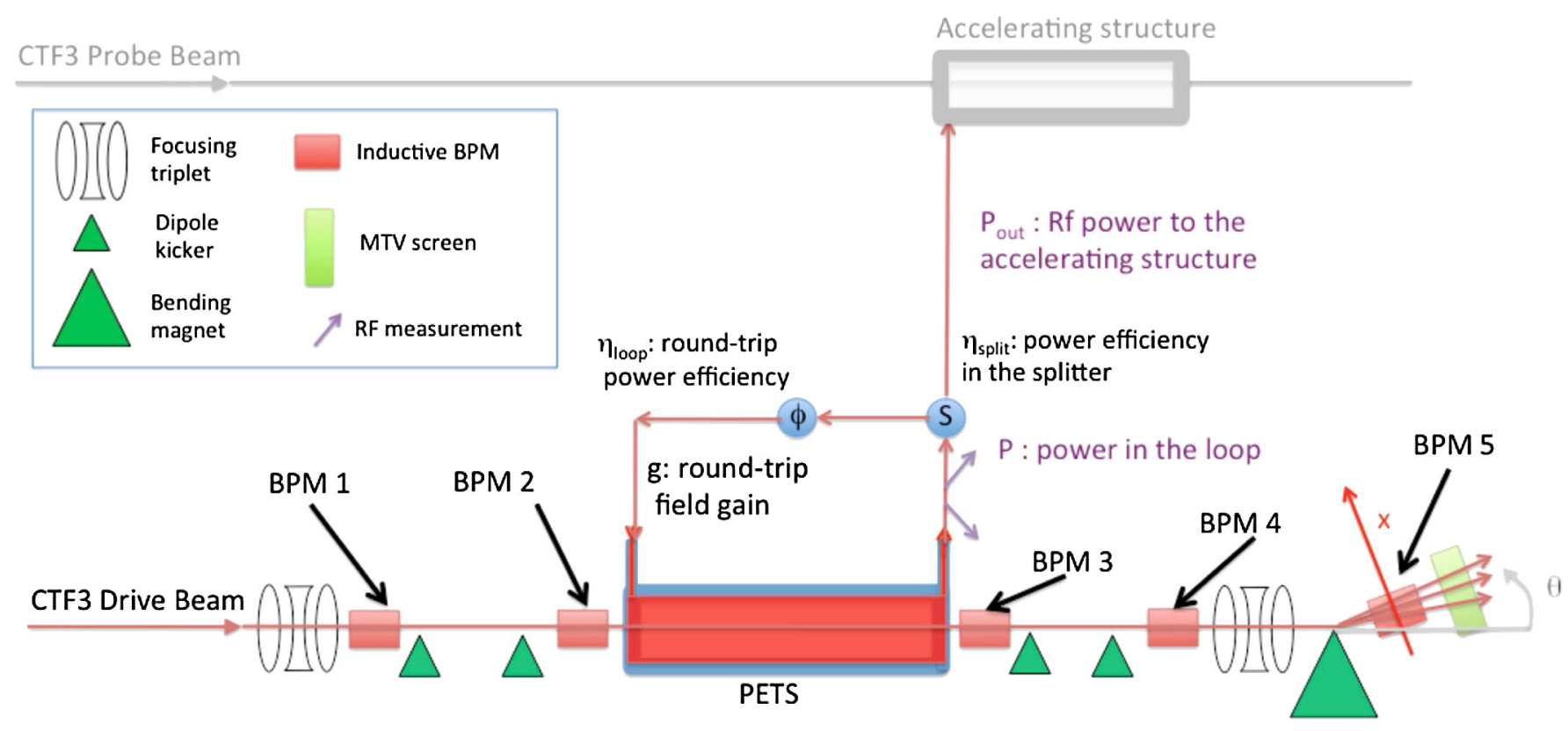

FIG. 2. Sketch of the drive beam line of the two-beam test stand. The drive beam is decelerated in the PETS, extracting rf power in the process. Part of the rf power is fed back using a recirculation loop, providing additional deceleration and power production for a given drive beam current. The drive beam line is equipped with inductive BPMs, rf windows, and a spectrometer dump. The rf power produced is used to power an $X$-band accelerating structures in the probe beam part, indicated in grey. 


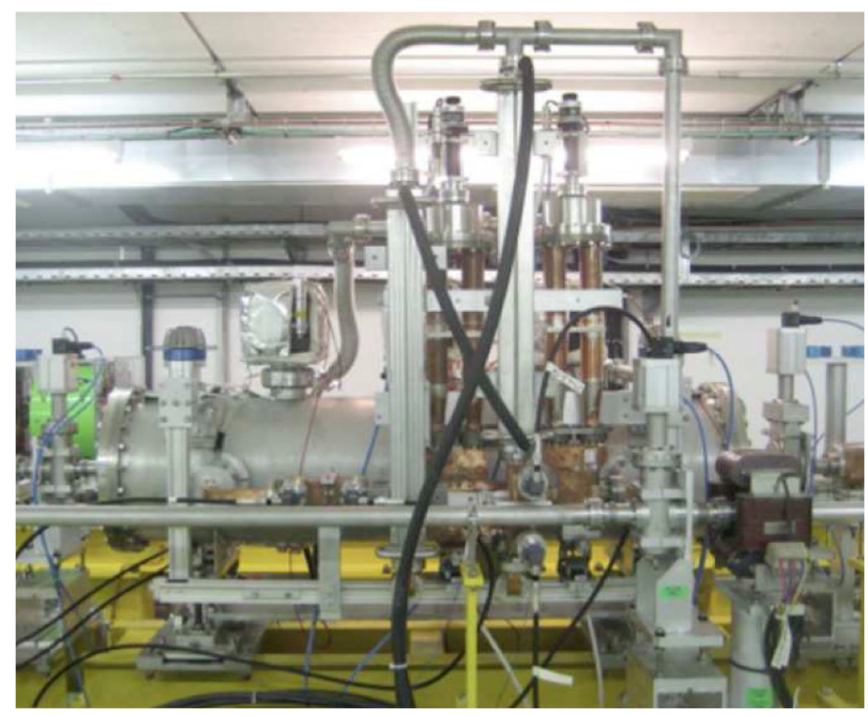

FIG. 3. The TBTS PETS tank with the power recirculator and the variable power splitter and phase shifter installed.

in a structure filled with the rf power produced by the PETS. The full TBTS setup is described in [3]. We are here concerned with the characterization of the power production and deceleration, and consider only the drive beam line. The TBTS drive beam line is equipped with $\mathrm{rf}$ measurement windows and inductive beam position monitors (BPMs) [6]. In this paper we make use of the BPM intensity (sum) signals of the BPM just after the PETS for power reconstruction, the BPM horizontal signals in the two BPMs upstream of the spectrometer dump for compensation of transverse motion, the spectrometer BPM signal, and the rf diode signal for the PETS output power. The measurements for the rf power outside the loop were not considered trustworthy during this run (problems with nonlinearities and broken electronics were reported), and are for this reason not used to compare the PETS output power rf signal. The TBTS drive beam setup, including the instrumentation used in this analysis, is illustrated in Fig. 2. Figure 3 shows the TBTS PETS tank with the power recirculator and the variable power splitter and phase shifter installed.

\section{PETS POWER PRODUCTION}

In the two-beam acceleration scheme, the rf power for the main beams is produced when the drive beam interacts with the impedance of the periodically loaded PETS structure, exciting preferentially the synchronous mode with frequency $\omega_{\mathrm{rf}} / 2 \pi=12 \mathrm{GHz}$. Extensive studies have been performed to arrive at the current CLIC PETS design, including studies of high-power behavior [7-9] and higherorder mode behavior $[7,10,11]$. The PETS prototype analyzed in this paper is the first $X$-band PETS tested with beam. The power produced in the constant impedance, high group-velocity PETS is given by $[7,11]$

$$
P_{\text {imp }}=\frac{1}{4}\left(R^{\prime} / Q\right) \frac{\omega_{\mathrm{rf}}}{v_{g}} L^{2} I^{2} F^{2} \eta_{\Omega}^{2},
$$

where $R^{\prime} / Q=2220$ linac $-\Omega / \mathrm{m}$ is the PETS impedance per meter, $v_{g}=0.46 c$ the group velocity, $L=1.0 \mathrm{~m}$ the PETS length, $\eta_{\Omega}^{2}=0.96$ the Ohmic loss efficiency, $I$ the drive beam current, and $F$ the beam form factor. We use the suffix "imp" to indicate power extracted due to the PETS impedance, without recirculation applied. PETS low power rf measurements of $S$-parameters and the synchronous frequency yield an expected power production efficiency of $99.6 \%$ with respect to the calculated [12]. For our analysis we consider all parameters except the current and the form factor as fixed.

In the TBTS, the power output from the PETS is split, in a ratio tunable by the operator, between the feedback arm and the arm out towards the probe beam. The total power efficiency in the splitter (including Ohmic losses and reflections) is measured to be $\eta_{\text {split }}=0.90 \pm 0.01 \%$. Of particular interest for our analyses is the total power efficiency for a full round-trip around the recirculation loop, including the splitter, measured to be

$$
\eta_{\text {loop }}=0.75 \pm 0.01 \% \text {. }
$$

We suggest a two-parameter model to describe the effect of the PETS recirculation loop. This model has been developed in [11,13] and has been applied earlier in $[11,13-16]$. The physics of the model is best presented using a field picture. The structure rf field, $E$, is in general related to the rf power in a structure, $P$, by $[7,11]$

$$
P=\frac{|E|^{2} v_{g}}{\left(R^{\prime} / Q\right) \omega_{\mathrm{rf}}}
$$

We define $g$ as the field round-trip gain around the recirculation loop and $\phi$ as the phase at which the recirculated field adds to the beam generated field. The field seen at the PETS output at a time step $m, E_{m}$, is the field seen one round-trip time earlier, $E_{m-1}$, plus the field generated by the beam interacting with the PETS impedance at this time, $E_{\text {imp,m }}[14]$,

$$
E_{m}=g e^{i \phi} E_{m-1}+E_{\mathrm{imp}, m} .
$$

All the physics in our two-parameter recirculation model is contained in Eq. (4). The gain is given by the ratio of the power splitted back into the PETS, $S$, and the total roundtrip efficiency, as

$$
g=\sqrt{S \eta_{\text {loop }}}
$$

The drive beam current entering the TBTS may vary both along the pulse and from pulse to pulse. We take into account an arbitrary beam current profile by calculating $E_{\text {imp }}$ as a function of the current $[13,14]$. Figure 4 shows an example of the predicted increase in power production due to recirculation, using the two-parameter model on a 


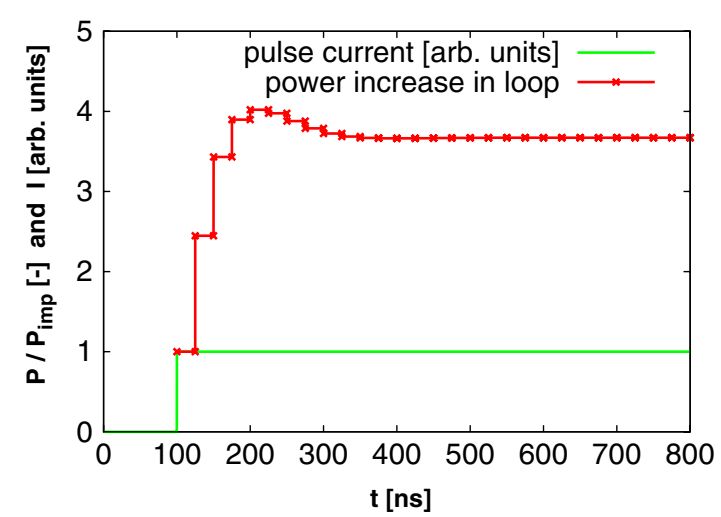

FIG. 4. Example of power production with recirculation. The figure shows the ratio of power in the recirculation loop to the power production without recirculation, for a rectangular power pulse entering the PETS at $t=100 \mathrm{~ns}$. The recirculation gain in the example is $g=0.6$ and the recirculation phase $\phi=25 \mathrm{deg}$. The recirculation loop round-trip time is $25 \mathrm{~ns}$. After about $200 \mathrm{~ns}$ a steady-state power level 3.7 times higher than without recirculation is achieved in the loop.

rectangular current pulse, with a gain of $g=0.6$ and a recirculation phase of $\phi=45 \mathrm{deg}$. The loop round-trip time, i.e., the time between time step $m$ and $m-1$, is estimated to $25 \mathrm{~ns}$. After $100 \mathrm{~ns}$ the drive beam pulse enters the PETS and produces power according to Eq. (1). After each recirculation round-trip time the total power in the loop is calculated by propagating the field according to Eq. (4) then applying Eq. (3). We observe that a steady-state (s.s.) power level is reached after a few hundred ns. The steady-state power level, $P_{\text {s.s. }}$, with respect to the power level without recirculation, $P_{\text {imp }}$, can be calculated by infinite summing of Eq. (4) then applying Eq. (3), yielding [14]

$$
P_{\text {s.s. }} / P_{\text {imp }}=\frac{1}{\left(1-2 g \cos \phi+g^{2}\right)} .
$$

It is of interest to calculate the total power exiting the PETS recirculation loop. We consider two contributions: $P_{\text {out }}$, the rf power exiting the loop in the direction of the probe beam indicated in Fig. 2, and $P_{\text {lost }}$, the power dissipated in the loop and the splitter (invisible losses). The PETS downstream choke is estimated to give an attenuation of about $50 \mathrm{~dB}$, so we assume that no rf power is leaking in the direction downstream of the beam line. A fraction $1-S$ of the intra-PETS power $P$ exits the loop and is attenuated by a factor $\eta_{\text {split }}$ yielding the power exiting the loop,

$$
P_{\text {out }}=\eta_{\text {split }}(1-S) P .
$$

The total power dissipated in the loop is the sum of the part that is dissipated in the splitter $\left(1-\eta_{\text {split }}\right)(1-S) P$ and the part dissipated from the recirculated fraction of the power $S P$ in the remainder of the loop $\left(1-\eta_{\text {loop }}\right) S P$. The total dissipated power is thus

$$
P_{\text {lost }}=\left(1-\eta_{\text {loop }}\right) S P+\left(1-\eta_{\text {split }}\right)(1-S) P .
$$

The total power exiting the recirculation loop is then the sum of $P_{\text {out }}$ and $P_{\text {lost }}$. Inserting the previous expressions leads, after simplifications and utilizing Eq. (5), to

$$
P_{\text {out }}+P_{\text {lost }}=\left(1-g^{2}\right) P .
$$

We will in the following use Eq. (7) to correlate measured power with the total beam energy loss.

The produced power depends strongly on the beam form factor which is proportional to the Fourier transform of the charge distribution and quantifies the reduction in power production due to finite bunch length and bunch phase errors. We discuss here briefly the reduction due to single bunch length and reduction due to bunch phase errors individually, as both effects are relevant for the TBTS power production. Assuming a Gaussian bunch with rms length $\sigma_{z}$, the single bunch form factor can be written $F_{\mathrm{SB}}=\exp \left(-\frac{1}{2} \sigma_{z} \omega_{\mathrm{rf}} / c\right)$. Various streak camera bunch length measurements have been performed in the combiner ring, yielding estimates of FWHM values from 15-30 ps $\left(\sigma_{z} \sim 2-4 \mathrm{~mm}\right)$ with the bunch length some times varying along the train [17]. Between the ring and the experimental area there are several horizontal and vertical chicanes and the optics here is not known with sufficient precision to extrapolate the bunch length measurements from the ring with confidence. Furthermore, if the drive beam bunches are not combined perfectly with $12 \mathrm{GHz}$ spacing, the power production will be reduced due to out-of-phase additions of the multibunch wakefields. In CTF3, phase variations along the train may be induced by energy variations in the linac, combined with a nonzero longitudinal dispersion from the linac to the rings. In CTF3 the $12 \mathrm{GHz}$ phase variation along the uncombined train has been measured to be in the order of $10^{\circ}$ after optimization, corresponding to a power reduction due to bunch phase errors in the order of $1 \%$ [18]. In the following we will regard the total form factor as an unknown parameter to be identified.

Our recirculation model does not take into account bunch length variations along the pulse, bunch phase errors, nor break down activity. For the measurements discussed in this paper, we operated at working points with very little break down activity and optimized klystron phases. We will later quantify the performance of the model by calculating the difference between measured and reconstructed power.

\section{MEASUREMENTS}

We analyze in this paper three data series containing a total of 500 pulses, all with the same CTF3 drive beam configuration, optimized for the power production characterization work. The $1.5 \mathrm{GHz}$ subharmonic bunchers were disabled and the delay loop was bypassed. The $3 \mathrm{GHz}$ beam entering the combiner ring was combined by a factor 4, generating a $12 \mathrm{GHz}$ drive beam entering CLEX. The 
drive beam energy was measured to be $\mathcal{E}_{\text {beam }}=113 \mathrm{MeV}$ (lower than the CTF3 design energy since two klystrons were not operative). The mean drive beam current measured in the TBTS was about $8.5 \mathrm{~A}$. The current pulse full length was about $300 \mathrm{~ns}$, with a flattop length of more than $200 \mathrm{~ns}$. There was noticeable pulse to pulse jitter, originating mainly from the energy variations in the linac, leading to jitter in both the incoming energy, the transverse phase space, the current, and subsequently also power and deceleration.

The measurement series each have a different setting of the PETS recirculation power splitter and within each series the phase shifter is varied over the full range. Figure 5 illustrates the measurement data for series 1 . The graphs show the average of all pulses in the series along the pulse. The mean and standard deviation of the average of the flattop of the pulse are also given. Figure 5(a) shows the sum signal in the BPM located just after the TBTS PETS. The mean current along the flattop part of the pulse is $-8.57 \pm 0.40 \mathrm{~A}$. Figure 5(b) shows the reading of the power output from the PETS with the phase shifter adjusted for zero phase shift. The mean power along the flattop part of the pulse is $75.4 \pm 3.9 \mathrm{MW}$.
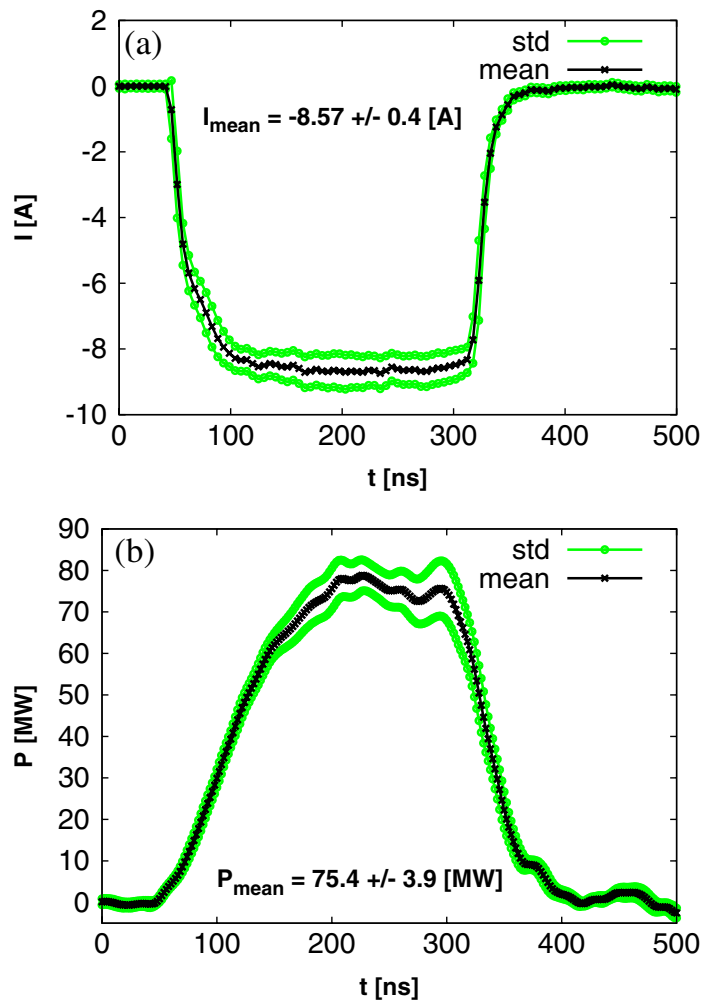

FIG. 5. Summary of the measurements data for series 1 . (a) Sum signal in the BPM just after the TBTS PETS. The mean along the flattop part of the pulse is $-8.57 \pm 0.40 \mathrm{~A}$. (b) Power output from the PETS with the phase shifter adjusted for zero phase shift. The mean along the flattop part of the pulse is $75.4 \pm 3.9 \mathrm{MW}$.

\section{ESTIMATION OF RECIRCULATION PARAMETERS}

To analyze the power production, a precise knowledge of the recirculation gain and phase is required. We here introduce a method to identify these parameters without relying on values extrapolated from shut-down measurements and control system settings. Assuming the twoparameter recirculation model, Eq. (6) yields the increase in steady-state power production due to the recirculation, $P_{\text {s.s. }}$, with respect to power production without recirculation, $P_{\text {imp }}$, as a function of the gain and phase. Figure 6(a) shows a plot of $P_{\text {s.s. }} / P_{\text {imp }}$ for gains of $g=0.5$ and $g=0.6$ as a function of the phase $\phi$. The width of the resonance peak does not depend on the absolute value of the measured power, and is thus not influenced by calibration errors. In order to identify the recirculation working point for a fixed splitter setting, we vary the phase and extract the steady-state part of the power pulse, normalize it to current, and fit the theoretical curve using three parameters, gain $g$, zero point for the phase $\phi_{0}$, and a constant scaling $c$ which
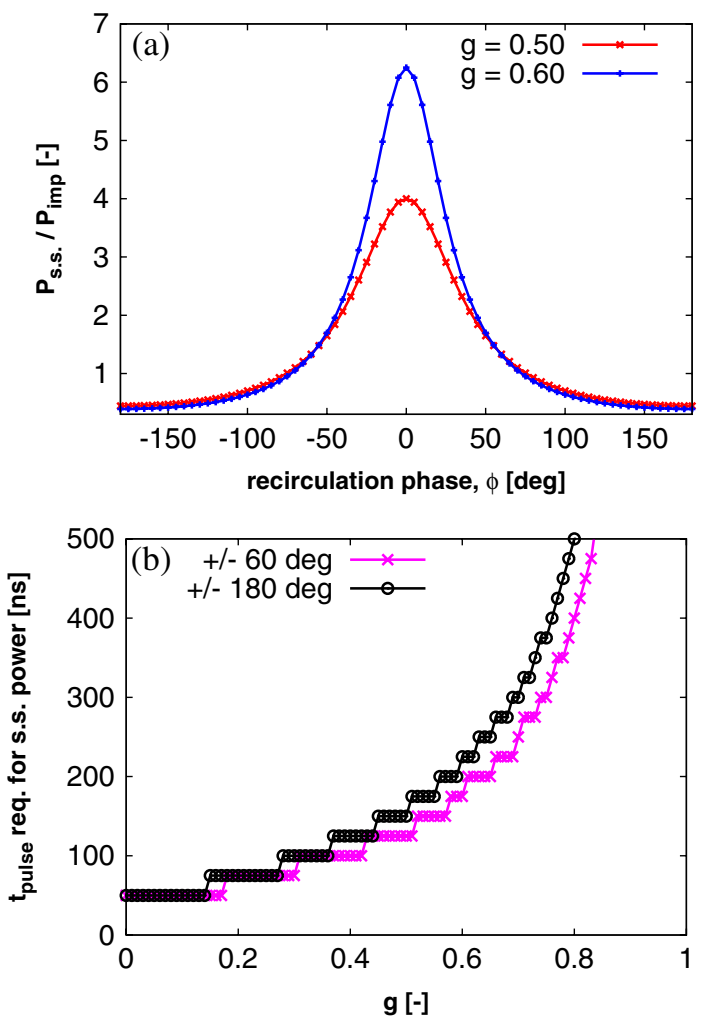

FIG. 6. (a) Theoretical steady-state power production with respect to power production without recirculation according to Eq. (6), as a function of recirculation phase for gain. $(\times)$ : 0.5 , (o): 0.6. (b) Current pulse length needed to reach steady-state power production, as a function of the recirculation gain. $(\times)$ : Steady state is reached for the whole phase range. (o): Steady state is reached for a phase range of $\pm 60 \mathrm{deg}$. For comparison Fig. 4 illustrates the evolution to steady state for a single setting of $g=0.6, \phi=25 \mathrm{deg}$. 
contains both the unknown form factor and potential calibration errors. This approach assumes we have reached near steady-state conditions for the recirculating power pulse. Using Eqs. (4) and (6) we calculate current pulse length required to reach steady-state conditions, taken to be a power difference from one round-trip to the next of less than $5 \%$. Figure $6(\mathrm{~b})$ shows the required pulse length as a function of the recirculation settings. The current for the measurements shown in Fig. 5(a) has a flattop length of slightly above $200 \mathrm{~ns}$, allowing steady-state conditions to be reached for gain settings up to $g \approx 0.6$, for all phase settings.

For a given splitter setting we have performed a full phase scan between extreme limits of the phase shifter stepper motor, and logged a number of pulses for each phase setting. The measured power is averaged over the last $20 \%$ of the pulse, and normalized to the pulse current squared. The measurements are then fitted to the curve
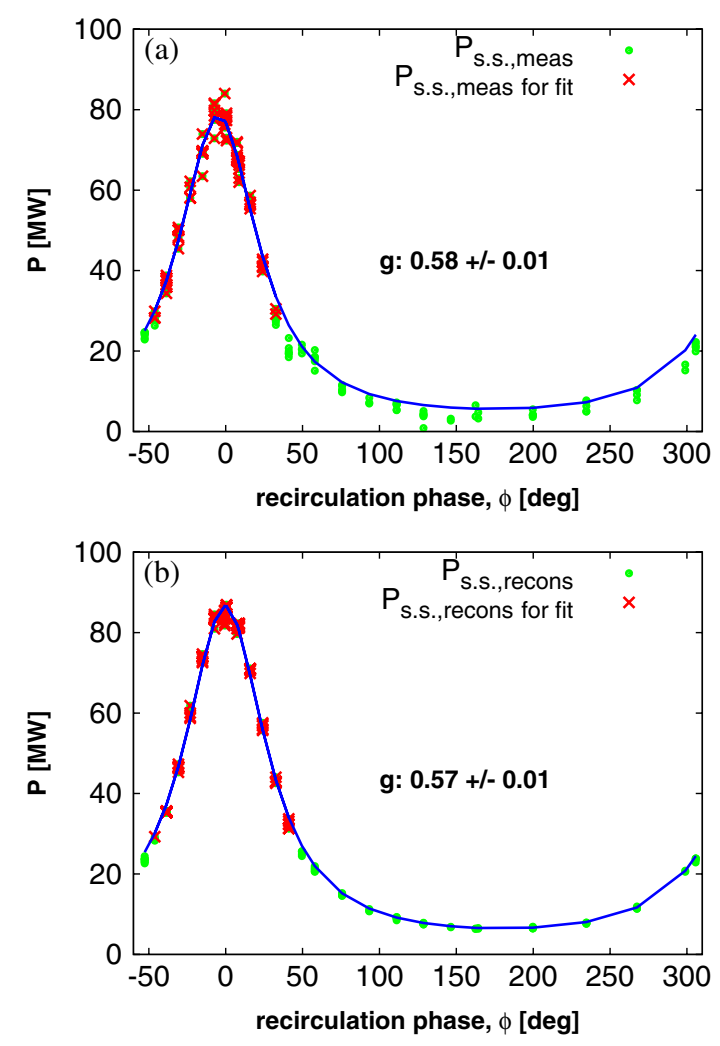

FIG. 7. (a) Measured power for a fixed power splitter setting, scanning the full phase range. $(X)$ : Measurements with power level down to $1 / 3$ of the maximum, used for fitting. (-): The curve described by Eq. (6). (o): The full range of power measurements, used to verify the scale of the phase shifter settings. (b) Validation of the phase scan method by reestimating the gain from power reconstructed from a given input gain. $(\times)$ : Reconstructed power with power level down to $1 / 3$ of the maximum, used for fitting. (-): The curve described by Eq. (6). (o): The full range of reconstructed power. The estimate for the reconstructed phase scan is correct within the fit error.
TABLE I. The middle column shows the estimated recirculation loop gain from the measurements. The last column shows the reestimated gain from the reconstructed measurement series. The gain estimates from the reconstructed phase scan are correct within the fit error.

\begin{tabular}{lcc}
\hline \hline Series \# & $g_{\text {est, meas }}$ & $g_{\text {est, reconstr }}$ \\
\hline 1 & $0.58 \pm 0.01$ & $0.57 \pm 0.01$ \\
2 & $0.62 \pm 0.01$ & $0.61 \pm 0.01$ \\
3 & $0.51 \pm 0.02$ & $0.50 \pm 0.01$ \\
\hline \hline
\end{tabular}

described by Eq. (6) using nonlinear optimization to fit the three parameters $g, \phi_{0}$, and $c$. Because of the noise being more dominant at low power levels, we fit the width of the curve from maximum to one-third of maximum. Figure 7(a) shows for series 2 the measurements used for the fit in red $(X)$ and the other measurements in green (o). The curve described by Eq. (6), with the fitted parameters, is shown in blue (一). The power values shown in Fig. 7 are scaled so that the peak normalized power corresponds to peak measured power $(85 \mathrm{MW})$. The commanded phase settings extends the full $360 \mathrm{deg}$ range, and no significant scale error in the logged phase splitter settings was observed.

The values of the gains calculated from the measured power, $g_{\text {est, meas }}$, are given in the second column of Table I, ranging from 0.51 to 0.62 . The calculated gains were initially found to be about $15 \%$ too high with respect to a gain estimate based on shut-down measurements together with logged values of splitter and phase shifter settings. Further offline analysis of the system showed that the identified and estimated gains were consistent when the temperature dependence of the splitter position is compensated for, since the temperature at the time of the shutdown measurements was different from the temperature during operation. The phase scan method has proven very useful to debug the system and to improve the precision of future TBTS data analysis, and will be used in future runs.

\section{POWER RECONSTRUCTION}

Using the identified gain and zero phase, we reconstruct the PETS power from the measured current for each pulse, applying our model according to Eqs. (3) and (4). We first apply the phase scan method described in the last section to the reconstructed power pulses in order to validate the method. Ideally we would recover the gain we used for the reconstruction. Figure 7(b) illustrates the phase scan applied to the reconstructed power for series 1 . The results of the gain identifications from the reconstructed power, $g_{\text {est, reconstr, }}$, are given in the last column of Table I. We observe that the phase scan method indeed estimates within the error the gain used for the reconstruction, $g_{\text {est, meas }}$.

We now estimate the form factor for each series by doing a global fit of the peak reconstructed power to the peak 


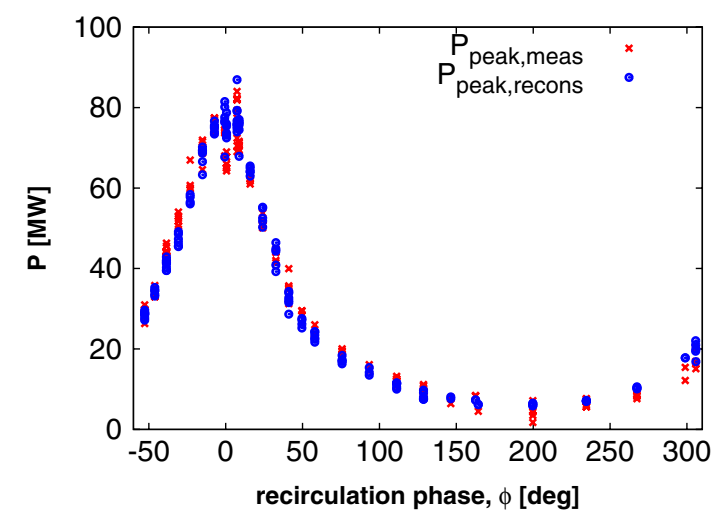

FIG. 8. Peak power for the reconstructed (o) and measured power $(\times)$, after the form factor has been fitted. The agreement is good over the full range of the measurement data.

measured power for each series. Because of the potential form factor variations within the measurement series both from pulse to pulse, and along pulses, the resulting estimate must be interpreted as an average form factor for each series. Using this method the preliminary form factor estimate for all series is $0.80 \pm 0.03$. Assuming perfect bunch phase and no calibration errors, this would correspond to an average rms bunch length of $2.4-2.8 \mathrm{~mm}$, for a Gaussian bunch. Figure 8 shows the measured and reconstructed peak power for measurement series 1 applying the estimated form factor for the reconstructed power. All the measured individual pulses in each series are regenerated using the same three identified parameters (gain, zero phase, and form factor). We observe that the agreement between measurement and model reconstruction is good over the full phase range. Figure 9 shows examples of individual pulses with four different values of the phase $\phi$. Figure 9(a) has zero recirculation phase and yields a peak power of $80 \mathrm{MW}$. In comparison, for this current level (about 8.5 A) and form factor, direct power production without recirculation would, according to Eq. (1), yield 14 MW. Figure 9(b) has a phase of $111 \mathrm{deg}$, yielding a destructive interference of the field. We note that this is reflected in the reconstructed as well as the measured power. Figures 9(c) and 9(d) have a recirculation phase of $23 \mathrm{deg}$ and $39 \mathrm{deg}$, respectively. According to the model the power decays after the end of the beam current pulse with a time constant $\tau$ given by the gain $g$ and the recirculation time $t_{r}$ as $\tau=-t_{r} / \ln \left(g^{2}\right)$ [14]. The measurement and the reconstruction slopes appear consistent giving additional indications that the gain has been correctly identified.

Figures 8 and 9 show that the two-parameter model preserves overall features of the produced power well; however, depending on the pulse the detailed structure may not be accurately represented, since break down
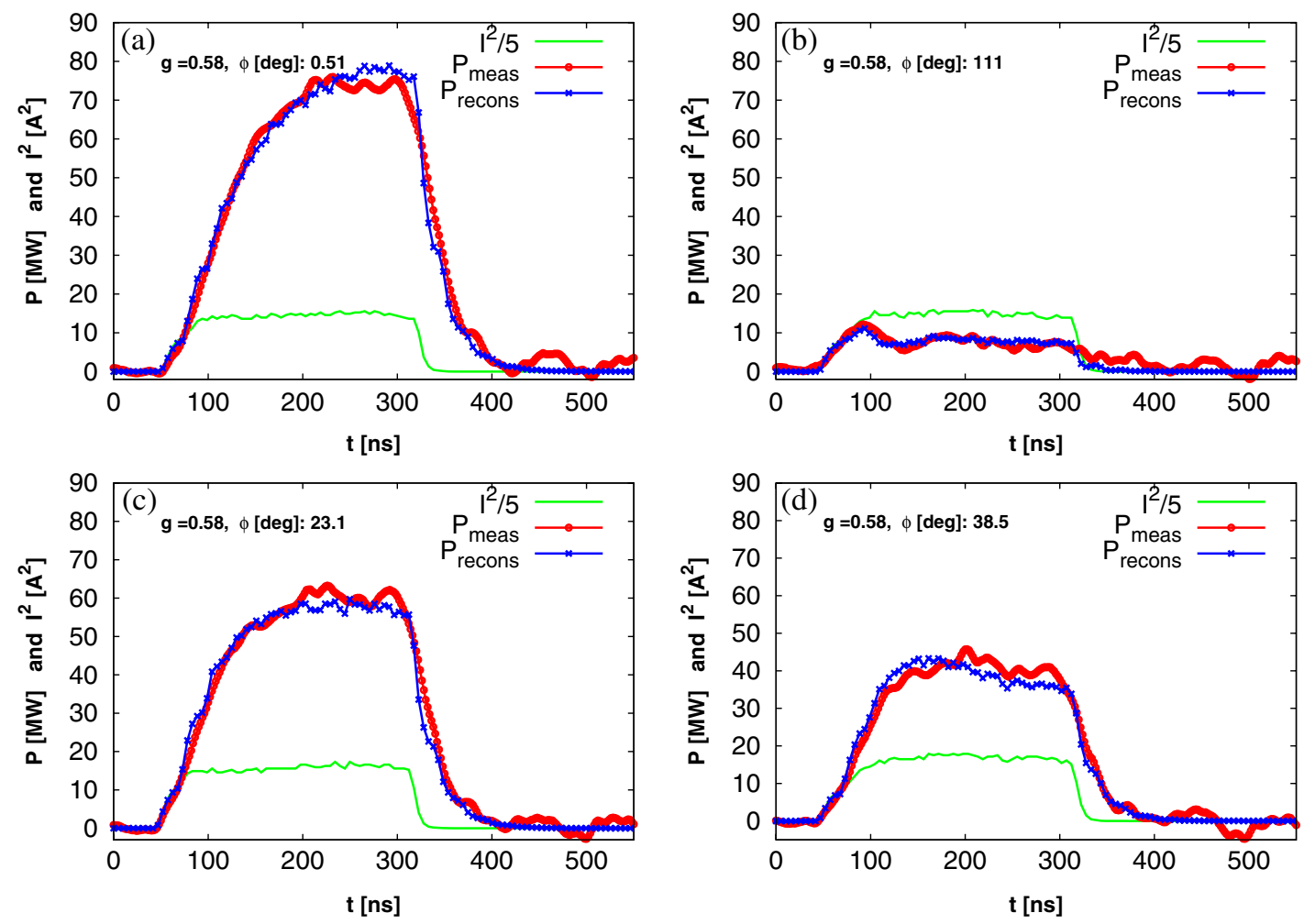

FIG. 9. Examples of power pulses for different recirculation phases. Measured power (o) and reconstructed power $(\times)$ are shown, together with the current pulse input for the reconstruction (-). (a) Zero recirculation phase, which yields a peak power of 80 MW. (b) Phase of $111 \mathrm{deg}$, yielding a destructive interference of the field. (c) Phase of $23 \mathrm{deg}$. (d) Phase of 39 deg. In comparison, the power level without recirculation would be about $14 \mathrm{MW}$. 
TABLE II. Maximum rms difference between reconstructed and measured power for $75 \%$ of the pulses. For all reconstructed pulses, about $75 \%$ of the pulses have an rms difference less than $15 \%$, indicating an overall reasonable agreement for the power reconstructed with a two-parameter model.

\begin{tabular}{lc}
\hline \hline Series \# & Maximum $\varepsilon_{P}$ for $75 \%$ of the pulses \\
\hline 1 & $13 \%$ \\
2 & $18 \%$ \\
3 & $15 \%$ \\
\hline \hline
\end{tabular}

activity, bunch length variations along the pulse, and bunch phase errors potentially produce discrepancies between reconstruction and measurements. As a metric for the correspondence between measured $\left(P_{\text {meas }}\right)$ and reconstructed power $\left(P_{\text {recons }}\right)$, we calculate the relative rms difference for each pulse,

$$
\varepsilon_{P}=\sqrt{\frac{\sum\left(P_{\text {recons }}-P_{\text {meas }}\right)^{2}}{\sum P_{\text {recons }}^{2}}},
$$

where the summation goes over all samples. To quantify the performance of the model we calculate the value for which the $\varepsilon_{P}$ of $75 \%$ of the pulses is smaller. The result is summarized in Table II. We use the $75 \%$ quartile to discard outlier pulses resulting from machine instabilities, typically temporary klystron malfunction, leading to abnormal bunch length and/or bunch phasing. For all reconstructed pulses, about $75 \%$ of the pulses have an rms difference less than $15 \%$, indicating an overall reasonable agreement for the deceleration reconstructed with a two-parameter model. $^{2}$ In comparison, in [15] $75 \%$ of the pulses were calculated to give an rms difference of less than $10 \%$; however, all the pulses were logged for a single splitter and phase setting because the splitter and phaser shifter were not remotely operable during that run. The results shown here are thus the first confirmation that the model presented is valid for a large range of splitter and phase shifter settings.

\section{DECELERATION}

We now turn to comparing the power generated in the PETS to the energy lost by the electron beam. First we consider the total energy balance and later we investigate the variation of the energy loss along the beam pulse. The power produced is extracted from the drive beam and the resulting beam deceleration is measured in the TBTS spectrometer dump. Figure 2 illustrates the setup used for the deceleration measurements. The horizontal position in the spectrometer BPM, $x$, located just after the dipole is used to measure the beam centroid deceleration. The spectrometer magnet bends the center momentum particle by an angle of $\theta=22.8^{\circ}$, yielding a dispersion of

\footnotetext{
${ }^{2}$ The pulses shown in Fig. 9 have an $\varepsilon_{P}$ from $6 \%$ to $13 \%$.
}

$D=0.23 \mathrm{~m}$ at the spectrometer BPM. For a beam entering the spectrometer at zero angle and offset, the BPM will measure a position $x=-D \delta_{\text {spec }}$, where $\delta_{\text {spec }}$ is the total momentum variation with respect to the center momentum. In general, the centroid momentum along the CTF3 drive beam pulses may vary along the pulse and jitter from pulse to pulse. The spectrometer measures the sum of the momentum variation entering the PETS, $\delta_{\text {incoming }}$, and the momentum variation due to the deceleration, $\delta_{\mathrm{PETS}}$,

$$
\delta_{\text {spec }}=\delta_{\text {PETS }}+\delta_{\text {incoming }} .
$$

In Eq. (8) we scale the relative incoming variation by the ratio of the centroid momentum before the PETS to the mean centroid momentum after PETS deceleration. To estimate the incoming energy variation we use one BPM before and two BPMs after a dog-leg upstream of the PETS allowing for separation of the effect of incoming angle from an energy variation, yielding the required $\delta_{\text {incoming }}$ [19]. Furthermore, the drive beam will enter the spectrometer at a nonzero angle and offset where the values may jitter from pulse to pulse. This angle and offset will be converted in a horizontal position in the spectrometer BPM, and must be compensated for, using the BPMs in front of the spectrometer magnet. The effect on the horizontal position in the spectrometer is found using transfer matrices derived from a MAD-X model [20]. Since the available measurement sets cover a wide range in power and deceleration, we disregard static uncertainties in the incoming beam parameters (offset, angle, incoming momentum) by looking only at relative energy loss from pulse to pulse. This approach has the substantial advantages that we do not need to take into account BPM and quadrupole misalignment, we do not need to take into account corrector settings and corrector calibrations, and we do not need to identify an absolute zero of the spectrometer readings. This approach thus removes several potential error sources. Calculating relative deceleration measurements still allows us to investigate correlations with power measurements, both from pulse to pulse and along the pulses, as will be explained in the following.

We analyze the correlation between deceleration and power measurements by considering the energy loss of the whole pulse as we consider this the most robust approach. Later, we complement this with studies of energy loss along single pulses. By energy conservation, for any pulse independent of the recirculation settings, the total energy lost out of the system PETS plus recirculation loop, denoted $E_{\text {pow }}$, should equal the total energy lost by the beam, denoted $E_{\text {spec }}$. We calculate the pulse energy lost out of the system as

$$
E_{\text {pow }}=\int\left(P_{\text {out }}+P_{\text {lost }}\right) d t,
$$

where $P_{\text {out }}+P_{\text {lost }}$ was derived in Eq. (7). The relative pulse beam energy loss we calculate as 


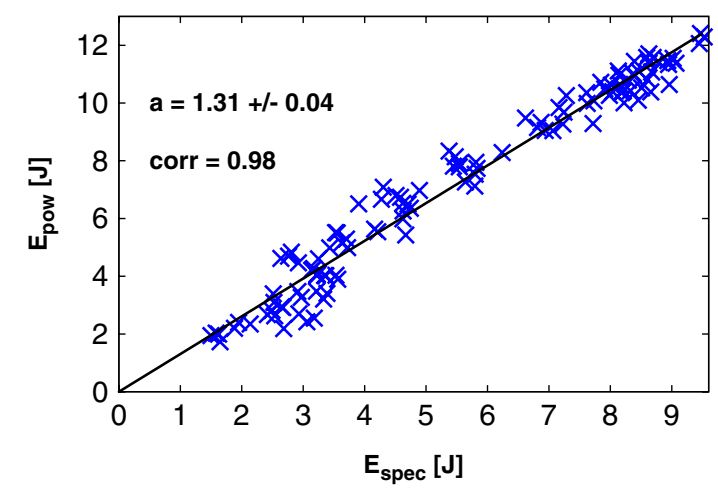

FIG. 10. Correlation of drive beam energy loss measured in the spectrometer $\left(E_{\mathrm{spec}}\right)$, and system energy loss deduced from power measurements $\left(E_{\text {pow }}\right)$. We observe the expected linear correlation, however, the estimated slope (1.3) is larger than expected (1).

$$
E_{\mathrm{spec}}^{R}=\int U_{\mathrm{spec}}^{R} I d t
$$

where $I$ is the drive beam current measured just after the PETS and $U_{\text {spec }}^{R}=\mathcal{E}_{\text {beam }} \delta_{\text {PETS }}^{R}$ is the relative centroid energy loss measured in the spectrometer.

We apply the pulse energy loss correlation for the three measurement series analyzed previously. Figure 10 illustrates the correlated energy loss for series 1. For each point on the graph, the ordinate is calculated using Eqs. (7) and (9), where the PETS power measurements are used together with the gain estimates in column two of Table I. The abscissa is calculated using Eq. (10) applying correction for jitter in incoming angle, position, and energy. The abscissa is shifted so that the zero point for $E_{\text {spec }}$ agrees with the zero point for $E_{\text {pow }}$, and the superscript " $R$ " is subsequently omitted from here. We observe, as expected, a linear correlation between $E_{\text {pow }}$ and $E_{\text {spec }}$ for all data series (the linear correlation for the series ranges from 0.97 to 0.99 ). We furthermore perform a linear leastsquare fit $E_{\text {pow }}=a E_{\text {spec }}+b$. The offset, $b$, will in general be nonzero, since the deceleration is calculated with respect to an unknown zero point. The slope, $a$, we expect to be close to identity. The results of the linear least-square estimation is given in Table III. The errors include the point spread and the error in the gain estimates. The reason that $b$ varies is that the spectrometer settings, including the zero point, were slightly retuned for each series.

We note that for all series we calculate a slope of about 1.3, instead of the expected 1. One explanation of the slope of 1.3 could be calibration errors in the measurements, possibly in the rf and/or in the BPM signals. The PETS output power, in the order of $100 \mathrm{MW}$, is attenuated by about $100 \mathrm{~dB}$ before measurement (in the form of couplers, cables, fixed and variable attenuators) and $1 \mathrm{~dB}$ error in the total rf calibration chain would lead to a power measurement error of 1.3. In the CLEX there has not been an
TABLE III. Linear correlation coefficient, slope $a$, and offset $b$ for each measurement series. The slope is consistently about a factor 1.3 higher than expected. The variation in the offset is due to the fact that the spectrometer settings were adjusted between each series.

\begin{tabular}{lccc}
\hline \hline Series \# & Linear correlation & $a$ & $b$ \\
\hline 1 & 0.98 & $1.31 \pm 0.04$ & $1.96+0.14$ \\
2 & 0.99 & $1.33 \pm 0.04$ & $1.33+0.09$ \\
3 & 0.97 & $1.34 \pm 0.05$ & $1.95+0.18$ \\
\hline \hline
\end{tabular}

independent cross-check of the rf calibration factors, and problems were reported with connectors and electronics at various points during this run. On the other hand, the CLEX drive beam BPMs have been operative since 2008 and have shown a good level of consistency. In principle, the method of correlating energy loss from deceleration and from rf power described in this paper could be used as an independent cross-check of rf calibration factors. Contributing the estimated slope to power rf calibration errors (i.e. the real power is $30 \%$ lower than the measured), the field form factors, as derived in Sec. VI, must according to Eq. (1) be multiplied by a factor $1 / \sqrt{1.3}$, yielding revised average form factor estimates of $0.70 \pm 0.04$ for the three series. Assuming perfect bunch phase and no calibration errors, this would correspond to an average rms bunch length of 3.1-3.6 mm, for a Gaussian bunch. In principle, the analysis methods described in this paper could be used as an independent cross-check of form factor measurements, and ultimately be used for the benchmarking of bunch length measurements.

Having considered the total power balance above, we now investigate the variation along the pulse by comparing the measured deceleration to deceleration calculations from power measurements. The required expression is most easily developed using power considerations. The power output from the PETS at recirculation time step $m$, $P_{m}$, must equal a fraction $g^{2}$ of power of the PETS power at the previous time step, plus the power extracted from the beam, $P_{\text {beam, } m}{ }^{3}$

$$
P_{m}=g^{2} P_{m-1}+P_{\text {beam }, m}
$$

In general, the power generated by the beam is related to the deceleration by

$$
P_{\text {beam }, m}=U_{\text {pow }, m} I_{m} \eta_{\Omega} \text {, }
$$

where $\eta_{\Omega}=0.98$ is the Ohmic efficiency, $I_{m}$ the beam current at time step $m$, and $U_{\text {pow, } m}$ the deceleration at time

\footnotetext{
${ }^{3}$ The beam can be both decelerated or accelerated depending on the phase shifter settings and $P_{\text {beam, } m}$ can be both positive (power is generated by beam) or negative (power is absorbed by the beam). In general, $P_{\text {beam }}$ differs from $P_{\text {imp }}$ in Eq. (1).
} 

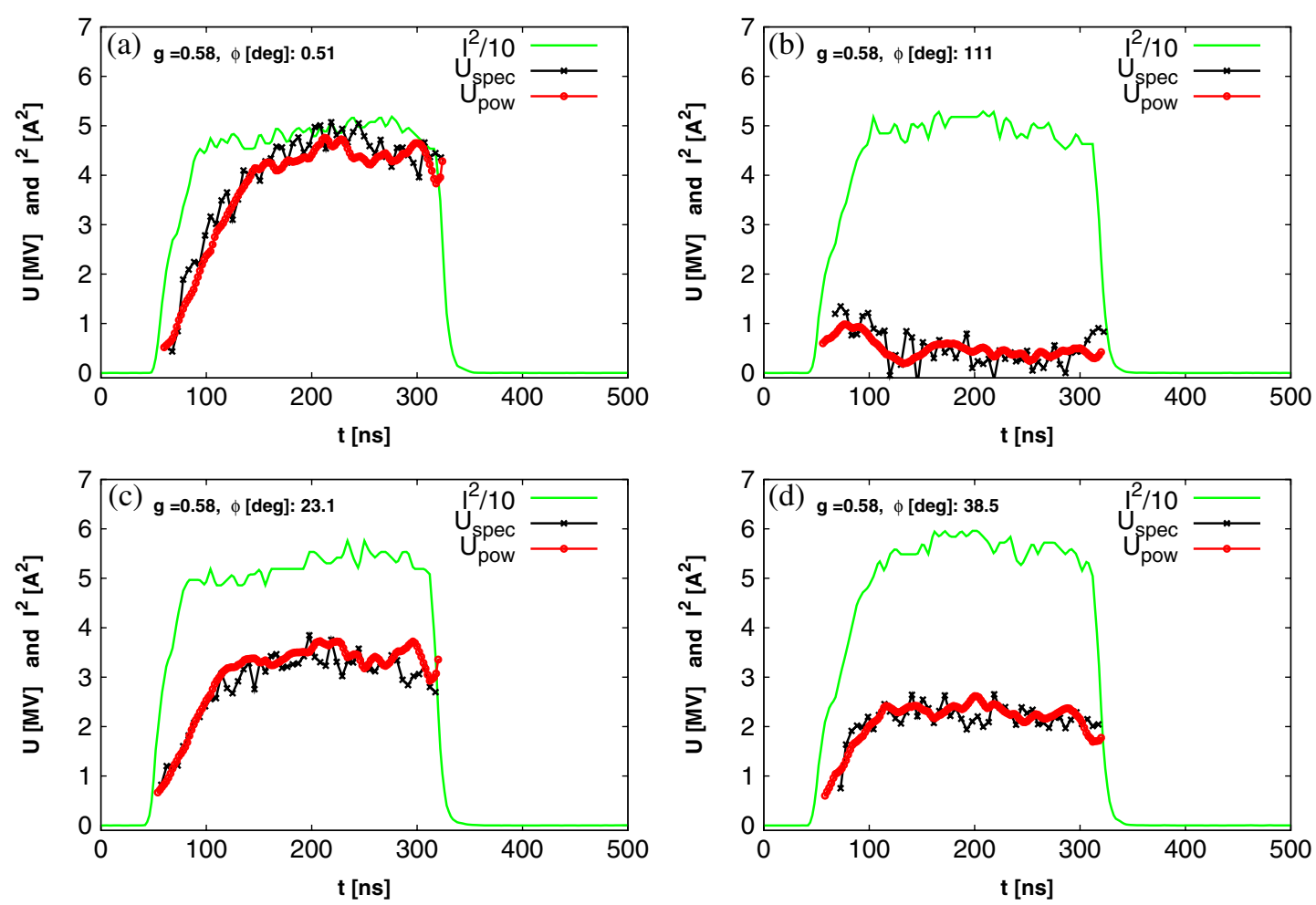

FIG. 11. Examples of the deceleration for different recirculation phases. Deceleration calculated with the spectrometer $(\times)$ and calculated from power measurements (o) are shown, together with the pulse current (-). (a) Recirculation phase of 0 deg. (b) Phase of $111 \mathrm{deg}$. (c) Phase of $23 \mathrm{deg}$. (d) Phase of $39 \mathrm{deg}$. This figure is to be compared to Fig. 9, showing the measured and reconstructed power for the same pulses.

step $m$. Combining Eqs. (11) and (12) yields the required estimate for the deceleration along the pulse, based on power and current measurement,

$$
U_{\text {pow }, m}=\left(P_{m}-g^{2} P_{m-1}\right) /\left(I_{m} \eta_{\Omega}\right)
$$

The measured deceleration, $U_{\text {spec }}$, for a series is offset to the deceleration as deduced by the power, $U_{\text {pow }}$, using Eq. (13) analogously to what was done for the energy loss. When comparing measured deceleration along the pulse with deceleration deduced from the power, we scale the power measurements by the estimated slope, $a$, identified in Table III. Figure 11 shows examples of pulses for different recirculation phases. The pulses are the same as presented in Fig. 9: Figure 11(a), recirculation phase of 0 deg; Fig. 11(b), phase of 111 deg; Fig. 11(c), phase of 23 deg; and Fig. 11(d), phase of 39 deg. The pulse shown in Fig. 11(b) is below the noise limit, defined as $0.5 \mathrm{MW}$, and is not taken into account in the correlation calculations shown in Fig. 10. It is shown here for consistency with Fig. 9. As an overall metric for the correspondence between deceleration measured with the spectrometer $\left(U_{\text {spec }}\right)$ and derived from the power measurements $\left(U_{\text {pow }}\right)$, we calculate the relative rms difference for each pulse,

$$
\varepsilon_{U}=\sqrt{\frac{\Sigma\left(U_{\text {spec }}-U_{\text {pow }}\right)^{2}}{\Sigma U_{\text {pow }}^{2}}},
$$

where the summation goes over all samples. We calculate the value for which the $\varepsilon_{U}$ of $75 \%$ of the pulses is smaller. The result is summarized in Table IV. For all reconstructed pulses, about $75 \%$ have an rms difference of less than $28 \%$, indicating a reasonable agreement for the deceleration reconstructed with a two-parameter model. In comparison, in [15] $75 \%$ of the pulses were calculated to give an rms difference of less than 20\%; however, all the pulses were logged for a single splitter and phase setting because the splitter and phase shifter were not remotely operable during that run. The results

TABLE IV. Maximum rms difference between reconstructed and measured deceleration for $75 \%$ of the pulses. For all reconstructed pulses, about $75 \%$ have a difference less than $28 \%$, indicating an overall reasonable agreement for the deceleration reconstructed with a two-parameter model.

\begin{tabular}{lc}
\hline \hline Series \# & Maximum $\varepsilon_{U}$ for $75 \%$ of the pulses \\
\hline 1 & $24 \%$ \\
2 & $32 \%$ \\
3 & $22 \%$ \\
\hline \hline
\end{tabular}


shown here validate the model based deceleration calculations for a large range of splitter and phase shifter settings.

\section{CONCLUSIONS}

We have characterized TBTS rf power extraction with recirculation by applying a two-parameter model where the field recirculation is characterized only by the gain and the recirculation phase. A method for identifying the recirculation parameters, based on performing a scan of the recirculation phase, has been developed. The twoparameter model using the identified system parameters gives a good fit between reconstructed power and measured power over a wide range of operational conditions. This has allowed for an overall estimation for the drive beam form factor.

Furthermore, we have calculated the drive beam deceleration along each pulse and have applied methods for deceleration calculations that are robust to the significant pulse jitter observed in the measurement data. Correlating the total pulse beam energy loss with the total system power loss we observe the expected linear correlations, though correlation coefficients indicate a possible calibration error of about $30 \%$ in the measurements signals.

Because of its simplicity, the model presented in this paper allows for analytical calculations of the expected power and deceleration for different drive beam and recirculation conditions. The proven validity of the model approach has increased substantially the understanding of the TBTS system. The model can therefore be used with confidence for estimations of future drive beam systems with field recirculation, such as those planned for upgrades of the CLIC Test Facility in the next phases of the project and towards the parameter regime of CLIC.

While this analysis is based on a two-parameter model for power extraction with field recirculation, increased agreement between the power measured and the power reconstructed may be achieved using a more complex model, taking into account bunch phasing, varying bunch form factor, and using a realistic impulse response.

\section{ACKNOWLEDGMENTS}

We acknowledge the support of the CTF3 operations team, F. Tecker, P. Skowronski, S. Bettoni, and J. Barranco, as well as the support of A.E. Dabrowski for beam diagnostics issues. We furthermore acknowledge useful discussions with D. Schulte, W. Wuensch, S. Doebert, and A. Palaia. This work is supported by the Research Council of Norway, the Swedish Research Council, and the Knut and Alice Wallenberg Foundation.

[1] G. Guignard et al. (The CLIC Study Team), Report No. CERN-2000-008, 2000.

[2] H. H. Braun et al., Report No. CERN-99-06, 1999.

[3] R. Ruber et al., in Proceedings of the 11th European Particle Accelerator Conference, Genoa, 2008 (EPS-AG, Genoa, Italy, 2008), p. 2821.

[4] G. Geschonke et al., CTF3 Design Report No. CERN-PS2002-008 (RF), 2002.

[5] S. Stapnes, Outlook on the next CLIC phase, 6th CLIC Advisory Committee (2011).

[6] M. Gasior, in Proceedings of the 6th European Workshop on Beam Diagnostics and Instrumentation for Particle Accelerators (GSI, Mainz, Germany, 2003), p. 53.

[7] I. Syratchev et al., in Proceedings of the 2007 Particle Accelerator Conference, Albuquerque, New Mexico (IEEE, New York, 2007), p. 2194.

[8] I. Syratchev et al., in Proceedings of the 11th European Particle Accelerator Conference, Genoa, 2008 (Ref. [3]), p. 1909.

[9] I. Syratchev et al., in Proceedings of the 23rd Particle Accelerator Conference, Vancouver, Canada, 2009 (IEEE, Piscataway, NJ, 2009), p. 1873.

[10] D. Schulte and I. Syratchev, in Proceedings of the 21st Particle Accelerator Conference, Knoxville, 2005 (IEEE, Piscataway, NJ, 2005), p. 1177.

[11] E. Adli, Ph.D. thesis, University of Oslo, 2009.

[12] I. Syratchev, Progress in the PETS development, CLIC09 Workshop (2009).

[13] R. Ruber and V. Ziemann, CERN CTF3 Note Report No. 092, 2009.

[14] E. Adli, CERN CTF3 Note Report No. 096, 2009.

[15] E. Adli et al., in Proceedings of the 19th European Workshop on Beam Diagnostics and Instrumentation for Particle Accelerators (PSI, Basel, Switzerland, 2009), p. 113.

[16] C. Hellenthal, Report No. CERN-OPEN-2010-010, 2010.

[17] A. E. Dabrowski et al. (unpublished).

[18] F. Tecker, Critical Review of the CTF3 Performance, International Workshop on Linear Colliders (2010).

[19] R. Ruber et al., CERN CTF3 Note Report No. 098, 2009.

[20] R. Ruber et al., MAD-X model for the Two-beam Test Stand (2009). 